Marc Via $\cdot$ Neus Valveny $\cdot$ Antonio López-Alomar

Georgios Athanasiadis · Xavier Pintó - Enric Domingo

Esther Esteban · Emili González-Pérez · Pedro Moral

\title{
E65 K polymorphism in KCNMB1 gene is not associated with ischaemic heart disease in Spanish patients
}

Received: 1 July 2005 / Accepted: 8 August 2005/Published online: 10 September 2005

(c) The Japan Society of Human Genetics and Springer-Verlag 2005

\begin{abstract}
Hypertension is a main risk factor for atherosclerosis through vascular wall hyperplasia. A recent study reported a new polymorphism $(\mathrm{E} 65 \mathrm{~K})$ in the $\beta_{1}$ subunit (KCNMB1) gene of the $\mathrm{Ca}^{2+}$-dependent potassium channel with a protective effect against the severity of diastolic hypertension, but further data have lead to conflicting results. In order to ascertain the involvement of the $\mathrm{E} 65 \mathrm{~K}$ variant in cardiovascular system regulation, the potential association between this mutation and ischaemic heart disease was assessed through a family-based association study $(n=302$ individuals). Transmission disequilibrium analysis failed to detect any association between this polymorphism and ischaemic heart disease. Although a minor effect cannot be discarded, sample analytical power and negative results do not support a major role for E65 K polymorphism in atherogenic pathologies.
\end{abstract}

Keywords Ischaemic heart disease $\cdot \mathrm{KCNMB} 1$ gene Polymorphism - Transmission disequilibrium test . Genetic risk

\section{Introduction}

Hypertension is a major chronic disease in developed countries and is a main cardiovascular risk factor. The

M. Via · N. Valveny · A. López-Alomar · G. Athanasiadis E. Esteban · E. González-Pérez · P. Moral $(\square)$

Unitat d'Antropologia, Departament de Biologia Animal,

Universitat de Barcelona, Barcelona, Spain

E-mail:pmoral@ub.edu

Tel.: + 34-93-4021461

Fax: + 34-93-4035740

X. Pintó

Unitat de Lípids i Arteriosclerosi, Hospital de Bellvitge, Spain

E. Domingo

Unitat Coronària-Secció d'Hemodinàmica,

Hospital Vall d'Hebron, Spain relationship between hypertension and atherosclerosis derives from the vascular smooth muscle hyperplasia induced by the increased vessel wall tension and also from the endothelial dysfunction caused by shear stress.

Genetic studies in animals and humans have revealed information about different pathways that contribute to genetic susceptibility to ischaemic heart disease (IHD), including lipoprotein metabolism, lipid oxidation, vascular tone, homocysteine regulation, and inflammation (for a review see Lusis et al 2004). Genes controlling vascular tone (nitric oxide synthase or adrenergic receptor genes) have been studied as candidate genes for cardiovascular disorders with different, often conflicting, results (Wang and Wang 2000; Naber et al 2004). Thus, many authors state that the natures of hypertension and cardiovascular disease are multifactorial, and hence multigenic (Doris 2002).

Vascular tone is controlled by a calcium signal in the vascular smooth muscle, and a key element of this is the large conductance $\mathrm{Ca}^{2+}$ and voltage-dependent $\mathrm{K}^{+}$ (BK) channel (Jaggar et al 2000). The BK channel consists of an ion-conducting $\alpha$ subunit and a regulatory $\beta_{1}$ subunit. Animal models have demonstrated that $\beta_{1}$ gene (KCNMB1) disruption results in increased arterial tone and blood pressure, leading to stroke, heart disease and renal disease (Brenner et al 2000).

Recently, an interesting association between a new single-nucleotide substitution (G352A) in the KCNMB1 gene and diastolic hypertension has been shown in a sample of Spanish subjects (Fernández-Fernández et al 2004). This mutation yields a glutamic acid-to-lysine change at codon 65 (E65 K). Functional findings together with epidemiological data lead the authors to the conclusion that the $\mathrm{K}$ allele has a protective effect against the severity of diastolic hypertension, suggesting that this mutation might offer a more efficient feedback mechanism for vascular smooth muscle contractility. Nevertheless, a very recent study has not been able to reproduce these results in a sample of 2130 Japanese hypertensive subjects (Kokubo et al 2005). 
In order to validate these observations and to test for a potential role of E65 K polymorphism in cardiovascular risk, we analyzed the possible association between this polymorphism and ischaemic heart disease (IHD) through a family-based association study. Altered vascular tone plays a major role in IHD, and a worthwhile hypothesis is that variations in diastolic pressure regulation efficiency may have an effect on cardiovascular disease aetiology (Nabel 2003). Besides, as far as we know, the possible relationship between KCNMB1 molecular variation and cardiovascular disease has never been tested.

\section{Materials and methods}

In this study, the E65 $\mathrm{K}$ polymorphism was analyzed in a sample of 101 nuclear families $(n=302)$. Each family consisted of both parents and IHD-affected offspring younger than 55 years. Family composition and recruitment criteria have been described previously (González-Pérez et al 2002). Written informed consent was obtained before a blood sample and data on cardiovascular risk factors were collected.

DNA was extracted by a phenol/chloroform procedure from the leukocyte fraction. E65 K polymorphism was analyzed using a Taqman assay (ABI PRISM 7900HT; Applied Biosystems, Foster City, CA, USA), using 5'-GCCACCTGATTGAGACCAACAT-3' and 5'-CCCACAGGCATGGGTACTG-3' primers, and VI C-ACCAGGAGGAGCTGA-MGB for the E ( $\mathrm{G}$ base) allele and FAM-ACCAGGAGAAGCTGA-MGB probe for the $\mathrm{K}$ (A base) allele.

Allelic and genotypic frequencies were computed by direct counting, and Hardy-Weinberg adjustment was assessed through Genetix 4.04 Software (available from http://www.univ-montp2.fr/ genetix/genetix/genetix.htm). The transmission disequilibrium test (TDT) was calculated using TRANSMIT software package (Clayton 1999). TDT evaluates the disequilibrium during allele transmission from heterozygous parents to affected offspring. The significance of the TDT values was re-assessed using permutation bootstrapping methods $(100,000$ iterations). Likewise, TDT estimates were also calculated by taking into account only unequivocal informative transmissions following the criteria established by Spielman et al (1993). Sample analytical power in TDT was calculated under a genotype relative risk $\gamma$ of 2.2 and a significance level $\alpha=0.05$ by TDT-PC software (Chen and Deng 2001). Under these assumptions, the sample size used here yielded an analytical power in TDT of $83.5 \%$.

\section{Results}

Genotypes for parents and offspring fitted HardyWeinberg equilibrium assumptions $(p=0.405$ and $p=0.907$, respectively). Allelic frequencies $(0.1037$ and 0.1224 for the $\mathrm{K}$ allele in parents and offspring, respectively) were similar to those previously described (Fernández-Fernández et al 2004; Kokubo et al 2005).

No transmission disequilibria were detected for any allele, not by TRANSMIT nor by Spielman criteria, as shown in Table 1. TDT values that resemble a chisquared distribution with one degree of freedom do not reach significance in any case. Moreover, this lack of association was confirmed when the whole sample was subdivided according to different classical environmental risk factors such as hypertension, smoking status, or gender.

\section{Discussion}

A hypothesis on the possible association between the E65 K polymorphism in the KCNMB1 gene and IHD risk has arisen due to recent results on the relationship between this polymorphism and the severity of diastolic hypertension (Fernández-Fernández et al 2004). Other studies, such as published associations between variants of the KCNMB1 gene and heart rate and baroreflex function seem to give additional indirect support to that hypothesis (Gollasch et al 2002). However, the present results do not confirm any relationship of this marker to the genetic susceptibility of IHD; rather they support the lack of association between E65 K and hypertension revealed by Kokubo et al (2005).

Table 1 TDT results for the K-allele in the whole sample and in different subsamples according to environmental factors

\begin{tabular}{|c|c|c|c|c|c|c|c|c|}
\hline & \multirow[t]{2}{*}{$N$} & \multicolumn{4}{|c|}{ TRANSMIT calculations } & \multicolumn{3}{|c|}{ Unequivocal transmissions } \\
\hline & & Obs $T$ & $\operatorname{Exp} T$ & $\chi^{2}$ & p-btp & Obs $T$ & $\operatorname{Exp} T$ & $\chi^{2}$ \\
\hline Total & 101 & 25 & 20.211 & 2.6829 & 0.1428 & 18 & 14.5 & 1.6897 \\
\hline Hypertension $^{\mathrm{a}}$ & 58 & 7 & 4.906 & 1.9767 & 0.2553 & 6 & 4 & 2.0000 \\
\hline Smoking ${ }^{\mathrm{b}}$ & 90 & 20 & 16.209 & 2.1009 & 0.1711 & 11 & 10 & 0.2000 \\
\hline Male sex & 83 & 22 & 17.984 & 2.1172 & 0.1844 & 13 & 11 & 0.7273 \\
\hline
\end{tabular}

Observed and expected transmissions, TDT values from TRANSMIT software, and unequivocal informative calculations are given. $p$ - $b t p$ bootstrap $p$-values for TRANSMIT calculations (100,000 bootstraps)

${ }^{a}$ Defined when individual has been diagnosed as being hypertense more than once before first IHD event (systolic pressure $\geq 160 \mathrm{mmHg}$ and/or diastolic pressure $\geq 95 \mathrm{mmHg}$ )

${ }^{\mathrm{b}}$ Smoker before first IHD event 
Taking into account the sample size (101 nuclear families) and the frequency of the $\mathrm{K}$ allele, a very low level of Type I and II error $(\alpha<0.05$ and power $>80 \%)$ can be maintained for a genotype relative risk equal to or greater than 2.2 (or a protective role equal to or lower than 0.46). We can rule out a significant effect of this polymorphism, but effects under this threshold cannot be rejected without a significant risk of committing a Type II error. Although minor effects cannot be discarded, a deep analysis of data in Table 1 indicates few trends consistent with any effect at all. If the $\mathrm{K}$ variant was a protective allele, we should expect lower observed transmissions than expected transmissions (although not reaching significant levels), but we obtained the reverse trend in our data.

To sum up, it is possible that the $\mathrm{E} 65 \mathrm{~K}$ polymorphism of the KCNMB1 gene might play a specific role only in severe diastolic hypertension, detectable from large samples such as those from Fernández-Fernández et al, but our data discard any etiologic relationship between this polymorphism and IHD. Further studies should focus on clarifying the potential role of this polymorphism in blood pressure regulation.

Acknowledgements This work was supported by the Dirección General de Investigación of the Ministerio de Ciencia y Tecnología (BMC2002-01224 project), and the Departament d'Universitats, Recerca i Societat de la Informació of the Generalitat de Catalunya (2001FI 00177 and 2002FI 00516 grants).

\section{References}

Brenner R, Pérez GJ, Bonev AD, Eckman DM, Kosek JC, Wiler SW, Patterson AJ, Nelson MT, Aldrich RW (2000) Vasoregulation by the betal subunit of the calcium-activated potassium channel. Nature 407:870-876
Chen WM, Deng HW (2001) A general and accurate approach for computing the statistical power of the transmission disequilibrium test for complex disease genes. Genet Epidemiol 21:53-67

Clayton D (1999) A generalization of the transmission disequilibrium test for uncertain-haplotype transmission. Am J Hum Genet 65:1170-1177

Doris PA (2002) Hypertension genetics, single nucleotide polymorphisms, and the common disease: common variant hypothesis. Hypertension 39:323-331

Fernández-Fernández JM, Tomás $\mathrm{M}$, Vázquez E, Orio $\mathrm{P}$, Latorre R, Senti M, Marrugat J, Valverde MA (2004) Gain-of-function mutation in the KCNMB1 potassium channel subunit is associated with low prevalence of diastolic hypertension. J Clin Invest 113:1032-1039

Gollasch M, Tank J, Luft FC, Jordan J, Maass P, Krasko C, Sharma AM, Busjahn A, Bahring S (2002) The BK channel betal subunit gene is associated with human baroreflex and blood pressure regulation. J Hypertens 20:927-933

González-Pérez E, Via M, López-Alomar A, Esteban E, Valveny N, Bao M, Domingo E, Moral P (2002) Lack of association between methylenetetrahydrofolate reductase (MTHFR) C677T and ischaemic heart disease (IHD): family based association study in a Spanish population. Clin Genet 62:235-239

Jaggar JH, Porter VA, Lederer WJ, Nelson MT (2000) Calcium sparks in smooth muscle. Am J Physiol Cell Physiol 278:C235C256

Kokubo Y, Iwai N, Tago N, Inamoto N, Okayama A, Yamawaki H, Naraba H, Tomoike H (2005) Association analysis between hypertension and CYBA, CLCNKB, and KCNMB1 functional polymorphisms in the Japanese population. Circ J 69:138-142

Lusis AJ, Mar R, Pajukanta P (2004) Genetics of atherosclerosis. Annu Rev Genomics Hum Genet 5:189-218

Naber CK, Siffert W, Erbel R, Heusch G (2004) Genetics of human coronary vasomotion. Arch Mal Coeur Vaiss 97:255-260

Nabel EG (2003) Cardiovascular disease. N Engl J Med 349:60-72

Spielman RS, McGinnis RE, Ewens WJ (1993) Transmission test for linkage disequilibrium: the insulin gene region and insulindependent diabetes mellitus (IDDM). Am J Hum Genet 52:506-516

Wang XL, Wang J (2000) Endothelial nitric oxide synthase gene sequence variations and vascular disease. Mol Genet Metab $70: 241-251$ 